\title{
ON A PROBLEM OF BARBAN AND VEHOV, CORRIGENDUM
}

\section{MATTI JUTILA}

Unfortunately the contours used in the proof of Lemma 3 of my recent paper [this volume pp. 62-71] have not all been specified in the appropriate way. The contours used at the foot of page 66 should be

$$
\begin{aligned}
& C_{0}: \quad \sigma=1 / \log x, \quad|t| \leqslant \exp \left((\log x)^{\frac{1}{2}}\right) ; \\
& C_{ \pm 1}: \quad 1 / \log x \leqslant \sigma \leqslant \alpha_{0}, \quad t= \pm \exp \left((\log x)^{\frac{1}{2}}\right) ; \\
& C_{ \pm 2}: \quad \sigma=\alpha_{0}, \quad|t| \geqslant \exp \left((\log x)^{\frac{1}{2}}\right) ;
\end{aligned}
$$

and middle contour used near the middle of page 67 should be

$$
C_{ \pm 1}: \quad c_{0} /(\log x)^{-\frac{1}{2}} \leqslant \sigma \leqslant 1 / \log x, \quad t= \pm \exp \left((\log x)^{\frac{1}{2}}\right)
$$

Department of Mathematics, University of Turku, Turku, Finland.
10H30: NUMBER THEORY; Multiplicative theory; Sieves.

Received on the 7th January, 1980. 